\title{
CHROMATIC PROPERTIES OF THE PANCAKE GRAPHS
}

\author{
Elena Konstantinova \\ Sobolev Institute of Mathematics, Novosibirsk, 630090, Russia \\ Novosibirsk State University, Novosibirsk, 630090, Russia \\ e-mail: e_konsta@math.nsc.ru
}

\begin{abstract}
Chromatic properties of the Pancake graphs $P_{n}, n \geqslant 2$, that are Cayley graphs on the symmetric group $S y m_{n}$ generated by prefix-reversals are investigated in the paper. It is proved that for any $n \geqslant 3$ the total chromatic number of $P_{n}$ is $n$, and it is shown that the chromatic index of $P_{n}$ is $n-1$. We present upper bounds on the chromatic number of the Pancake graphs $P_{n}$, which improve Brooks' bound for $n \geqslant 7$ and Catlin's bound for $n \leqslant 28$. Algorithms of a total $n$-coloring and a proper $(n-1)$-coloring are given.
\end{abstract}

Keywords: Pancake graph, Cayley graphs, symmetric group, chromatic number, total chromatic number.

2010 Mathematics Subject Classification: 05C15, 05C25, 05 C69.

\section{INTRODUCTION}

The Pancake graph $P_{n}=\left(\operatorname{Sym}_{n}, P R\right), n \geqslant 2$, is the Cayley graph on the symmetric group $S_{y m}$ of permutations $\pi=\left[\pi_{1} \pi_{2} \cdots \pi_{n}\right]$ written as strings in oneline notation, where $\pi_{i}=\pi(i)$ for any $1 \leqslant i \leqslant n$, with the generating set $P R=\left\{r_{i} \in \operatorname{Sym}_{n}: 2 \leqslant i \leqslant n\right\}$ of all prefix-reversals $r_{i}$ inversing the order of any substring $[1, i], 2 \leqslant i \leqslant n$, of a permutation $\pi$ when multiplied on the right, i.e., $\left[\pi_{1} \cdots \pi_{i} \pi_{i+1} \cdots \pi_{n}\right] r_{i}=\left[\pi_{i} \cdots \pi_{1} \pi_{i+1} \cdots \pi_{n}\right]$.

It is a connected vertex-transitive $(n-1)$-regular graph without loops and multiple edges of order $n$ !. The graph $P_{n}$ is almost pancyclic $[8,11]$ since it contains all cycles $C_{l}$ of length $l$, where $6 \leqslant l \leqslant n$ !, but does not contain cycles of length 3,4 or 5. This graph is well known because of the open combinatorial Pancake problem of finding its diameter [6].

A mapping $c: V(\Gamma) \rightarrow\{1,2, \ldots, k\}$ is called a proper $k$-coloring of a graph $\Gamma=(V, E)$ if $c(u) \neq c(v)$ whenever the vertices $u$ and $v$ are adjacent. The 
chromatic number $\chi(\Gamma)$ of a graph $\Gamma$ is the least number of colors needed to color vertices of $\Gamma$. A subset of vertices assigned to the same color forms an independent set, i.e., a $k$-coloring is the same as a partition of the vertex set into $k$ independent sets. The chromatic index $\chi^{\prime}(\Gamma)$ of a graph $\Gamma$ is the least number of colors needed to color edges of $\Gamma$ such that no two adjacent edges share the same color. In the total coloring of a graph $\Gamma$ it is assumed that no adjacent vertices, no adjacent edges, and no edge and its endvertices are assigned the same color. The total chromatic number $\chi^{\prime \prime}(\Gamma)$ of a graph $\Gamma$ is the least number of colors needed in any total coloring of $\Gamma$.

In this paper we prove the following result on the total chromatic number.

Theorem 1. $\chi^{\prime \prime}\left(P_{n}\right)=n$ for any $n \geqslant 3$.

The chromatic index of the Pancake graphs is obtained from Vizing's bound $\chi^{\prime} \geqslant \Delta[13]$ taking into account the edge coloring, in which the color $(i-1)$ is assigned to the prefix-reversal $r_{i}, 2 \leqslant i \leqslant n$ :

$$
\chi^{\prime}\left(P_{n}\right)=n-1 \text { for any } n \geqslant 3 .
$$

Since $P_{n}, n \geqslant 2$, is an $(n-1)$-regular graph, then by Brooks' theorem [2], stating that $\chi(\Gamma) \leqslant \Delta(\Gamma)$ for any connected graph $\Gamma$ with the maximum degree $\Delta=$ $\Delta(\Gamma)$, except for two cases, complete graphs and odd cycles, we have $\chi\left(P_{n}\right) \leqslant n-1$ for any $n \geqslant 3$. Let us note that $\chi\left(P_{3}\right)=2$ since $P_{3} \cong C_{6}$, and $\chi\left(P_{4}\right)=3$ since there are 7 -cycles in $P_{n}, n \geqslant 4$.

Thus, the trivial lower and upper bounds on the chromatic number of the Pancake graphs are given as follows:

$$
3 \leqslant \chi\left(P_{n}\right) \leqslant n-1 \text { for any } n \geqslant 4 .
$$

Borodin and Kostochka [1] showed that the Brooks' bound is improved by 1 for graphs with $\omega \leqslant(\Delta-1) / 2$, where $\omega$ is the size of the maximum clique in the graph. The same result was obtained independently by Catlin [3]. Since $\omega\left(P_{n}\right)=2$, then $\chi\left(P_{n}\right) \leqslant n-2$ for any $n \geqslant 6$. Moreover, there is a proper 3 -coloring of $P_{5}$. An example of such a proper 3-coloring for $P_{5}$ is presented in Appendix, where vertices $\left[\pi_{1} \pi_{2} \cdots \pi_{n-1} \pi_{n}\right]$ and $\left[\pi_{n} \pi_{n-1} \cdots \pi_{2} \pi_{1}\right]$ are assumed to be connected by an edge corresponding to $r_{n}$. Thus, finally we have

$$
\chi\left(P_{n}\right) \leqslant n-2 \text { for any } n \geqslant 5 .
$$

The Catlin's bound for $C_{4}$-free graphs [4], that is $\chi \leqslant \frac{2}{3}(\Delta+3)$, gives us $\chi\left(P_{n}\right) \leqslant$ $\frac{2}{3}(n+2)$ for any $n \geqslant 8$. The asymptotic bound $\chi\left(P_{n}\right) \leqslant O\left(\frac{n-1}{\log (n-1)}\right)$ follows from the Johansson result [7] for triangle-free graphs.

The following bounds on the chromatic number of the Pancake graphs are obtained in the paper. 
Theorem 2. The following hold for $P_{n}$ :

(i) if $5 \leqslant n \leqslant 8$, then

$$
\chi\left(P_{n}\right) \leqslant \begin{cases}n-k, & \text { if } n \equiv k(\bmod 4) \text { for } k=1,3 ; \\ n-2, & \text { if } n \text { is even; }\end{cases}
$$

(ii) if $9 \leqslant n \leqslant 16$, then

$$
\chi\left(P_{n}\right) \leqslant \begin{cases}n-(k+2), & \text { if } n \equiv k(\bmod 4) \text { for } k=1,3 \\ n-4, & \text { if } n \text { is even; }\end{cases}
$$

(iii) if $n \geqslant 17$, then

$$
\chi\left(P_{n}\right) \leqslant \begin{cases}n-(k+4), & \text { if } n \equiv k(\bmod 4) \text { for } k=1,2,3 \\ n-8, & \text { if } n \equiv 0(\bmod 4) .\end{cases}
$$

The bounds obtained improve (2) for $n \geqslant 7$ and Catlin's bound for $n \leqslant 28$. The paper is organized as follows. Theorem 1 is proved and an algorithm of total $n$-coloring is presented in Section 2. An algorithm of proper $(n-1)$-coloring of $P_{n}, n \geqslant 3$, is given in Section 3 and Theorem 2 is proved in Section 4 .

\section{Total $n$-COLORING}

The total coloring of a graph assumes that no adjacent vertices, no adjacent edges, and no edge and its endvertices are assigned the same color. The least number of colors needed in any total coloring of a graph $\Gamma$ gives its total chromatic number $\chi^{\prime \prime}(\Gamma)$. It is evident that $\chi^{\prime \prime}(\Gamma) \geqslant \Delta(\Gamma)+1$, so $\chi^{\prime \prime}\left(P_{n}\right) \geqslant n$.

The main result of this section is a proof of existence of a total $n$-coloring of $P_{n}, n \geqslant 3$, which is based on efficient dominating sets in the graph.

An independent set $D$ of vertices in a graph $\Gamma$ is an efficient dominating set if each vertex not in $D$ is adjacent to exactly one vertex in $D$. There are $n$ efficient dominating sets in $P_{n}[5,9,12]$ given by

$$
D_{k}=\left\{\left[k \pi_{2} \cdots \pi_{n}\right], \pi_{j} \in\{1, \ldots, n\} \backslash\{k\}, 2 \leqslant j \leqslant n\right\}, 1 \leqslant k \leqslant n .
$$

From (6) it follows that $\left|D_{k_{1}} \cap D_{k_{2}}\right|=0$, where $1 \leqslant k_{1} \leqslant n, 1 \leqslant k_{2} \leqslant n$, $k_{1} \neq k_{2}$, hence $n$ ! vertices of $P_{n}$ are colored by $n$ colors such that $c(\pi)=k$ for any $\pi \in D_{k}, 1 \leqslant k \leqslant n$.

The total $n$-coloring of $P_{n}$ is based on its hierarchical structure, which can be presented as follows. For any $n \geqslant 3$ the graph $P_{n}$ is constructed from $n$ copies of $P_{n-1}(i), 1 \leqslant i \leqslant n$, where each $P_{n-1}(i)$ has the vertex set $V_{i}=$ $\left\{\left[\pi_{1} \cdots \pi_{n-1} i\right]\right.$, where $\left.\pi_{k} \in\{1, \ldots, n\} \backslash\{i\}: 1 \leqslant k \leqslant n-1\right\},\left|V_{i}\right|=(n-1)$ !, 
and the edge set $E_{i}=\left\{\left\{\left[\pi_{1} \cdots \pi_{n-1} i\right],\left[\pi_{1} \cdots \pi_{n-1} i\right] r_{j}\right\}: 2 \leqslant j \leqslant n-1\right\}$, $\left|E_{i}\right|=\frac{(n-1) !(n-2)}{2}$. Any two copies $P_{n-1}(i), P_{n-1}(j), i \neq j$, are connected by $(n-2)$ ! edges $\left\{\left[i \pi_{2} \cdots \pi_{n-1} j\right],\left[j \pi_{n-1} \cdots \pi_{2} i\right]\right\}$, where

$$
\left[i \pi_{2} \cdots \pi_{n-1} j\right] r_{n}=\left[j \pi_{n-1} \cdots \pi_{2} i\right] .
$$

Prefix-reversals $r_{j}$, for $2 \leqslant j \leqslant n-1$, define internal edges in all $n$ copies $P_{n-1}(i)$, and the prefix-reversal $r_{n}$ defines external edges $E_{\text {ex }}$ between copies. Copies $P_{n-1}(i)$ are also called $(n-1)$-copies.

From (6) and from the hierarchical structure of $P_{n}$ it follows that efficient dominating sets of copies $P_{n-1}(i)$ are presented by all permutations with the first and the last elements fixed, namely

$$
D_{k}^{i}=\left\{\left[k \pi_{2} \cdots \pi_{n-1} i\right], \pi_{j} \in\{1, \ldots, n\} \backslash\{k, i\}, 2 \leqslant j \leqslant n\right\},
$$

where $1 \leqslant k \leqslant n, 1 \leqslant i \leqslant n, k \neq i$. There are $n(n-1)$ such sets of cardinality $(n-2)$ !. The sets defined by (6) and (8) admit the following obvious relationship:

$$
D_{k}=\bigcup_{i=1, i \neq k}^{n} D_{k}^{i} \text { for any } k=1, \ldots, n \text {. }
$$

Let us consider the following sets of edges:

$$
E_{i, j}=\left\{\{\pi, \tau\} \in E\left(P_{n}\right): \pi \in D_{i}, \tau \in D_{j}\right\}
$$

where $1 \leqslant i \leqslant n, 1 \leqslant j \leqslant n, i \neq j$,

$$
E_{i, j}^{k}=\left\{\{\pi, \tau\} \in E\left(P_{n}\right): \pi \in D_{i}^{k}, \tau \in D_{j}^{k}\right\}
$$

where $1 \leqslant i \leqslant n, 1 \leqslant j \leqslant n, 1 \leqslant k \leqslant n, i \neq j, i \neq k, j \neq k$, and $D_{i}, D_{j}$ are presented by (6), and $D_{i}^{k}, D_{j}^{k}$ are presented by (8). Since $P_{n}$ is an undirected graph, $E_{i, j}=E_{j, i}$. Sets given by (10) and (11) have independent edges, however sets $E_{i_{1}, j_{1}}$ and $E_{i_{2}, j_{2}}$ as well as sets $E_{i_{1}, j_{1}}^{k}$ and $E_{i_{2}, j_{2}}^{k}$ may have adjacent edges in $P_{n}$ if and only if $\left|\left\{i_{1}, j_{1}\right\} \cap\left\{i_{2}, j_{2}\right\}\right|=1$. We use this fact when colors are assigned to edges in $P_{n}$.

We assign colors for vertices and edges of $P_{n}$ from the set $\{0, \ldots, n-1\}$ as follows:

(a) if $n$ is odd then

$$
\left\{\begin{array}{l}
c\left(D_{k}\right)=2 k(\bmod n), \\
c\left(E_{i, j}\right)=i+j(\bmod n),
\end{array}\right.
$$

where $1 \leqslant i<j \leqslant n, 1 \leqslant k \leqslant n, i \neq k, j \neq k$; 
(b) if $n$ is even then

$$
\left\{\begin{array}{l}
c\left(D_{k}^{\ell}\right)=2(k-|k>\ell|+\ell)(\bmod (n-1)), \\
c\left(E_{i, j}^{\ell}\right)=i+j-|i>\ell|-|j>\ell|+2 \ell(\bmod (n-1)), \\
c\left(E_{e x}\right)=n-1,
\end{array}\right.
$$

where $1 \leqslant i<j \leqslant n, 1 \leqslant k \leqslant n, 1 \leqslant \ell \leqslant n, i \neq \ell, j \neq \ell, k \neq \ell$, and $|p|=1$, if $p$ is truly, and $|p|=0$, otherwise.

Proof of Theorem 1. Let us show that (12) and (13) give a total $n$-coloring of $P_{n}$ for any $n \geqslant 3$. We give a proof by a contradiction, assuming that such a coloring is not a total coloring, which means that some adjacent vertices, or adjacent edges, or an edge and its endvertices are assigned the same color.

If $n$ is odd, there exist $i, j, k$, where $1 \leqslant i \leqslant n, 1 \leqslant j \leqslant n, 1 \leqslant k \leqslant n, i \neq j$, $i \neq k, j \neq k$, such that one of the following equalities holds:

$$
c\left(D_{i}\right)=c\left(D_{j}\right), c\left(D_{i}\right)=c\left(E_{i, j}\right), c\left(E_{i, j}\right)=c\left(E_{i, k}\right) .
$$

Let us show that this is not true. Indeed, if there exist $i, j, i \neq j$, such that $c\left(D_{i}\right)=c\left(D_{j}\right)$, then $2 i \equiv 2 j(\bmod n)$ by $(12)$. Since $n$ is odd, this is possible only when $i=j$, which contradicts to the initial conditions. If $c\left(D_{i}\right)=c\left(E_{i, j}\right)$, then $2 i \equiv i+j(\bmod n)$ by $(12)$, which means that $i=j$ and we again have a contradiction. If $c\left(E_{i, j}\right)=c\left(E_{i, k}\right)$, then $i+j \equiv i+k(\bmod n)$ by (12) and we have $j=k$, but this is also not true. Hence, our assumption was wrong, and an $n$-coloring of the graph $P_{n}$ given by (12) is total.

If $n$ is even, then first we show that each of copies $P_{n-1}(\ell), 1 \leqslant \ell \leqslant n$, of the graph $P_{n}, n \geqslant 3$, has a total coloring given by (13). Let us assume that this is not true, i.e., there exist $i, j, k$, where $1 \leqslant i \leqslant n, 1 \leqslant j \leqslant n, 1 \leqslant k \leqslant n, i \neq j$, $i \neq k, i \neq \ell, j \neq k, j \neq \ell, k \neq \ell$, such that one of the following equalities holds:

$$
c\left(D_{i}^{\ell}\right)=c\left(D_{j}^{\ell}\right), c\left(D_{i}^{\ell}\right)=c\left(E_{i, j}^{\ell}\right), c\left(E_{i, j}^{\ell}\right)=c\left(E_{i, k}^{\ell}\right) .
$$

Let us check the first equality. If there exist $i, j, \ell$, where $1 \leqslant i \leqslant n, 1 \leqslant j \leqslant n$, $1 \leqslant \ell \leqslant n, i \neq j, i \neq \ell, j \neq \ell$, such that $c\left(D_{i}^{\ell}\right)=c\left(D_{j}^{\ell}\right)$, then $i-|i>\ell| \equiv j-\mid j>$ $\ell \mid(\bmod (n-1))$, but this leads to a contradiction on the initial conditions. Indeed, if $i>j>\ell$ or $i<j<\ell$, then $i=j$, which is not true. If $j<\ell<i$, then $i-1=j$, which is also not true, since $i<j$. If $i<\ell<j$, then $i=j-1$, which contradicts to $i+1<j$. The other two equalities are checked in the same way.

Thus, each of copies $P_{n-1}(\ell), 1 \leqslant \ell \leqslant n$, of the graph $P_{n}$ has a total coloring.

The external edges $E_{e x}$ of $P_{n}$ are colored by (13) with a color $(n-1)$. These edges are incident to vertices from efficient dominating sets $D_{i}^{j}$ and $D_{j}^{i}$ having different colors. Assume that $c\left(D_{i}^{j}\right)=c\left(D_{j}^{i}\right)$ for some $i, j, i \neq j, 1 \leqslant i \leqslant n$, $1 \leqslant j \leqslant n$. Then $i-|i>j|+j \equiv j-|j>i|+i(\bmod (n-1))$, and hence $0 \equiv 1(\bmod (n-1))$, which holds only for $n=2$. Thus, formulas $(12)$ and $(13)$ give a total coloring of the graph $P_{n}$ for any $n \geqslant 3$, and hence $\chi^{\prime \prime}\left(P_{n}\right)=n$. 
Table 1. Efficient dominating sets of $(n-1)$-copies of $P_{n}$

\begin{tabular}{|c|c|c|c|c|c|c|c|c|}
\hline & $P_{n-1}(1)$ & $P_{n-1}(2)$ & $\ldots$ & $P_{n-1}(i)$ & $\ldots$ & $P_{n-1}(j)$ & $\ldots$ & $P_{n-1}(n)$ \\
\hline$P_{n-1}(1)$ & - & $D_{2}^{1}$ & $\ldots$ & $D_{i}^{1}$ & $\ldots$ & $D_{j}^{1}$ & $\ldots$ & $D_{n}^{1}$ \\
\hline$P_{n-1}(2)$ & $D_{1}^{2}$ & - & $\ldots$ & $D_{i}^{2}$ & $\ldots$ & $D_{j}^{2}$ & $\ldots$ & $D_{n}^{2}$ \\
\hline$\ldots$ & $\ldots$ & $\ldots$ & - & $\ldots$ & $\ldots$ & $\ldots$ & $\ldots$ & $\ldots$ \\
\hline$P_{n-1}(i)$ & $D_{1}^{i}$ & $D_{2}^{i}$ & $\ldots$ & - & $\ldots$ & $D_{j}^{i}$ & $\ldots$ & $D_{n}^{i}$ \\
\hline$\ldots$ & $\ldots$ & $\ldots$ & $\ldots$ & $\ldots$ & - & $\ldots$ & $\ldots$ & $\ldots$ \\
\hline$P_{n-1}(j)$ & $D_{1}^{j}$ & $D_{2}^{j}$ & $\ldots$ & $D_{i}^{j}$ & $\ldots$ & - & $\ldots$ & $D_{n}^{j}$ \\
\hline$\ldots$ & $\ldots$ & $\ldots$ & $\ldots$ & $\ldots$ & $\ldots$ & $\ldots$ & - & $\ldots$ \\
\hline$P_{n-1}(n)$ & $D_{1}^{n}$ & $D_{2}^{n}$ & $\ldots$ & $D_{i}^{n}$ & $\ldots$ & $D_{j}^{n}$ & $\ldots$ & - \\
\hline
\end{tabular}

3. Proper $(n-1)$-COLORING

Efficient dominating sets (6) and (8) give us a simple algorithm of a proper $(n-1)$-coloring for $n$ ! vertices of $P_{n}, n \geqslant 3$. It follows from (7) and (8) that

$$
D_{j}^{i} r_{n}=D_{i}^{j}
$$

for any $i \neq j, 1 \leqslant i \leqslant n, 1 \leqslant j \leqslant n$, where $X r_{n}=Y$ means $x r_{n}=y$ for any $x \in X, y \in Y$, i.e., external edges between any two copies $P_{n-1}(i)$ and $P_{n-1}(j)$ of $P_{n}$ are incident to vertices from the sets $D_{j}^{i}$ and $D_{i}^{j}$ of corresponding copies as indicated by their superscripts.

These relationships between $(n-1)$-copies of the graph are shown in Table 1, where the entry in the $i$-th row and $j$-th column is referred to as the set $D_{j}^{i}$ of a copy $P_{n-1}(i)$ in $P_{n}$, whose vertices are adjacent to vertices of a copy $P_{n-1}(j)$ by the external edges. The union of all entries in the $i$-th row gives the vertex set of a copy $P_{n-1}(i)$, i.e., $\bigcup_{j=1, j \neq i}^{n} D_{j}^{i}=V\left(P_{n-1}(i)\right)$, and the union of all entries in the $j$-th column gives the efficient dominating set $D_{j}$, since by (9) we have $\bigcup_{i=1, i \neq j}^{n} D_{j}^{i}=D_{j}$. Moreover, the union of all sets (6) gives the vertex set of $P_{n}$.

Thus, on the one hand we have

$$
\bigcup_{i=1, j=1, i \neq j}^{n} D_{j}^{i}=V\left(P_{n}\right) \text { for any } n \geqslant 3,
$$

and on the other hand we have

$$
\bigcap_{i=1, j=1, i \neq j}^{n} D_{i}^{j}=0,
$$

which means that a proper $(n-1)$-coloring of all sets $(8)$ is a proper $(n-1)$ coloring for the graph at all. Below we present an algorithm for such a coloring. We write $c(X)=c$ when $c(x)=c$ for any $x \in X$. 


\section{Algorithm $P C$.}

Step 1. Set $c\left(D_{j}^{1}\right)=j-1$ for every $2 \leqslant j \leqslant n$.

Step 2. For every $2 \leqslant i \leqslant n$ and every $2 \leqslant j \leqslant n$, where $j \neq i$, set

$$
c\left(D_{j}^{i}\right)= \begin{cases}c\left(D_{j+1}^{i-1}\right)+1(\bmod (n-1)), & \text { if } i \neq j+2, \\ c\left(D_{j+2}^{i-2}\right)+1(\bmod (n-1)), & \text { if } i=j+2 .\end{cases}
$$

Theorem 3. Algorithm PC gives a proper $(n-1)$-coloring of $P_{n}$, for $n \geqslant 3$.

Proof. The following properties correspond to a proper $(n-1)$-coloring of $P_{n}$ : (a) vertices of the sets $D_{j}^{i}$ and $D_{i}^{j}, i \neq j$, given by relationship (14) must have different colors, i.e., $c\left(D_{j}^{i}\right) \neq c\left(D_{i}^{j}\right)$ for any $i, j \in\{1, \ldots, n\}$;

(b) vertices of any $(n-1)$-copy must have a proper $(n-1)$-coloring.

Let us show that Algorithm $P C$ holds these conditions. Indeed, Step 1 assigns colors from 1 up to $(n-1)$ to all sets $D_{j}^{1}, 2 \leqslant j \leqslant n$, and Step 2 assigns a color $c\left(D_{1}^{j}\right)=c\left(D_{j}^{1}\right)+1(\bmod (n-1))$ for each set $D_{1}^{j}$ by (15). Similarly, from (15) for any $i<j$ we get

$$
c\left(D_{i}^{j}\right)=c\left(D_{j}^{i}\right)+1(\bmod (n-1)),
$$

i.e., vertices of any two different efficient dominating sets of $(n-1)$-copies connected by external edges have different colors, hence property a) holds.

The property (b) holds since by (15) for a given $i, 2 \leqslant i \leqslant n$, sets $D_{j}^{i}$ get different $(n-1)$ colors for different $j, 2 \leqslant j \leqslant n, j \neq i$, i.e., vertices of any $(n-1)$-copy have a proper $(n-1)$-coloring. Let us note that vertices of the same set $D_{j}=\bigcup_{i=1, i \neq j}^{n} D_{j}^{i}$ may have the same color since they are at distance at least three from each other, by the definition. Thus, properties (a) and (b) hold, so Algorithm $P C$ gives a proper $(n-1)$-coloring of the graph $P_{n}$.

\section{Upper Bounds FOR $\chi\left(P_{n}\right)$}

Upper bounds on the chromatic number of the Pancake graph in Theorem 2 are obtained using the following properties of prefix-reversals.

Lemma 4. The prefix-reversal $r_{i}, 2 \leqslant i \leqslant n$, is even if and only if either $i \equiv$ $0(\bmod 4)$, or $i \equiv 1(\bmod 4)$.

Proof. Let us consider prefix-reversals $r_{2 k}$ and $r_{2 k+1}$, where $1 \leqslant k \leqslant \frac{(n-1)}{2}$, as a product of $k$ transpositions:

$$
\left\{\begin{array}{l}
r_{2 k}=(12 k)(22 k-1) \cdots(k k+1) \\
r_{2 k+1}=(12 k+1)(22 k) \cdots(k k+2) .
\end{array}\right.
$$

From (16) we immediately conclude that prefix-reversal $r_{i}$ is even if and only if either $i \equiv 0(\bmod 4)$, or $i \equiv 1(\bmod 4)$. 
Lemma 5. There is no expression for any prefix-reversal with even indexing as a product of prefix-reversals with odd indexing.

Proof. As one can see from (16), any prefix-reversal $r_{i}, 2 \leqslant i \leqslant n$, indexing by even $i$, changes parity of each elements of a substring $[1, i]$, but this does not hold for prefix-reversals with odd indexing.

Lemma 6. The Pancake graph $P_{n}, n \geqslant 3$, has $\frac{n !}{\ell}$ independent even $\ell$-cycles, where $6 \leqslant \ell \leqslant 2 n$.

Proof. It is evident that there are at most $\frac{n !}{\ell}$ independent $l$-cycles in a graph with $n$ ! vertices, so we have to describe such cycles for $\ell=2 k$, where $k \geqslant 3$. Let us consider cycles presented as product of $\ell$ prefix-reversals:

$$
C_{\ell}=r_{k} r_{k-1} \cdots r_{k} r_{k-1}=\left(r_{k} r_{k-1}\right)^{k}
$$

and let us show that they correspond to the lemma's conditions. Indeed, if $\pi=\left[\pi_{1} X\right]$, where $X=\pi_{2} \cdots \pi_{n}$, then $\pi r_{\ell} r_{\ell-1}=\left[\pi_{1} X\right] r_{\ell} r_{\ell-1}=\left[X \pi_{1}\right]$, and therefore, $\pi\left(r_{\ell} r_{\ell-1}\right)^{\ell}=\left[\pi_{1} X\right]\left(r_{\ell} r_{\ell-1}\right)^{\ell}=\left[\pi_{1} X\right]$. Moreover, since any vertex of $P_{n}$ is incident to exactly one edge corresponding to $r_{i}$, for any $2 \leqslant i \leqslant n$, hence the vertex must belong to the only cycle (17) and this completes the proof.

Let us note that Lemma 6 is a particular case of Lemma 1 from [10].

Proof of Theorem 2. We consider the set $E$ of all even prefix-reversals. From Lemma 4 we have $E=\left\{r_{i}, 2 \leqslant i \leqslant n: i \equiv 0(\bmod 4)\right.$ or $\left.i \equiv 1(\bmod 4)\right\}$. Then

$$
|E|= \begin{cases}\frac{n-k}{2}, & \text { if } n \equiv k(\bmod 4) \text { for } k=1,3 \\ \frac{n-2}{2}, & \text { if } n \text { is even. }\end{cases}
$$

Indeed, if $n$ is even, then there are exactly $\frac{n}{2}$ elements $i \in\{1, \ldots, n\}$ such that either $i \equiv 0(\bmod 4)$, or $i \equiv 1(\bmod 4)$, hence there are exactly $\frac{n-2}{2}$ such elements $i$, when $i \in\{2, \ldots, n\}$. Other cases in (18) are proved in the same way.

Now let us consider $E$-induced subgraphs of $P_{n}$ on either even or odd permutations with edge sets defined by the set $E$. These subgraphs form two sets $P_{n}[E]^{e}$ and $P_{n}[E]^{o}$, where $P_{n}[E]^{e}$ contains all $E$-induced subgraphs on even permutations, and $P_{n}[E]^{o}$ contains all $E$-induced subgraphs on odd permutations.

These sets have the following trivial properties.

1. All subgraphs from $P_{n}[E]^{e}$ and $P_{n}[E]^{o}$ are $|E|$-regular.

2. There are no edges between vertices of subgraphs of $P_{n}[E]^{e}\left(P_{n}[E]^{o}\right)$ in $P_{n}$.

3. Vertices of all subgraphs from $P_{n}[E]^{e}$ and $P_{n}[E]^{o}$ form the vertex set of $P_{n}$.

4. There are edges between vertices of subgraphs from $P_{n}[E]^{e}$ and $P_{n}[E]^{o}$ in $P_{n}$, moreover, these edges correspond to the odd prefix-reversals.

Then, by Brooks' theorem and property 1 , we have $\chi\left(P_{n}[E]^{e}\right) \leqslant|E|$ and $\chi\left(P_{n}[E]^{o}\right) \leqslant|E|$, and by properties $2-4$ we immediately have $\chi\left(P_{n}\right) \leqslant 2|E|$, 
where $|E|>2$, because otherwise odd cycles may appear. Let us show that this does not happen. Indeed, we have $|E|=2$ when $n=5,6,7$, so there are only two even prefix-reversals, namely, $r_{4}$ and $r_{5}$. Then, by Lemma 6 there are $n ! / 10$ independent 10-cycles presented by $\left(r_{4} r_{5}\right)^{5}$ in $P_{n}$ (see (17)), and hence, odd cycles do not appear. This means that

$$
\chi\left(P_{n}\right) \leqslant 2|E| \text { for any } n \geqslant 5 .
$$

Bound (3) in Theorem 2 follows from (18) and (19).

Since $\omega\left(P_{n}\right)=2$, Borodin-Kostochka's bound holds for $P_{n}[E]^{e}$ and $P_{n}[E]^{o}$ independently when $|E| \geqslant 4$, and hence it holds for $n \geqslant 9$. From this we immediately have $\chi\left(P_{n}\right) \leqslant 2(|E|-1)$ for any $n \geqslant 9$, which gives (4) in Theorem 2 .

Now let us consider the set $F=\left\{r_{4 i+1}, 5 \leqslant 4 i+1 \leqslant n\right\}$ of prefix-reversals with odd indexing whose cardinality is

$$
|F|= \begin{cases}\frac{n-k}{4}, & \text { if } n \equiv k(\bmod 4) \text { for } k=1,2,3 \\ \frac{n-4}{4}, & \text { if } n \equiv 0(\bmod 4) .\end{cases}
$$

Applying similar arguments and taking into account Lemma 5, we get $\chi\left(P_{n}\right) \leqslant$ $4|F|$ for any $n \geqslant 5$ and $\chi\left(P_{n}\right) \leqslant 4(|F|-1)$ for any $n \geqslant 17$, which gives (5) in Theorem 2 and completes the proof.

\section{Discussions: Exact Values of the Chromatic Number}

Thus, we have $\chi\left(P_{3}\right)=2$ since $P_{3} \cong C_{6}$, and $\chi\left(P_{4}\right)=3$ since there are 7 -cycles in $P_{n}, n \geqslant 4$. An example of a proper 3-coloring of $P_{5}$ is presented in Appendix. An optimal 4-coloring of $P_{6}$ was computed by Tomaž Pisanski, University of Primorska, and Jernej Azarija, University of Ljubljana, so $\chi\left(P_{6}\right)=4$. Moreover, since $P_{n-1}$ is an induced subgraph of $P_{n}$, then $\chi\left(P_{7}\right)$ is at least 4 , and from (3) we have $\chi\left(P_{7}\right)=4$. If $n=8$ then from (3) we have $4 \leqslant \chi\left(P_{8}\right) \leqslant 6$, and from (4) we have $4 \leqslant \chi\left(P_{n}\right) \leqslant 6$, where $9 \leqslant n \leqslant 16$. However, a proper 4 -coloring in these cases is unknown.

\begin{tabular}{|c|c|c|c|c|c|c|c|c|c|c|c|c|c|c|}
\hline$n$ & 3 & 4 & 5 & 6 & 7 & 8 & 9 & 10 & 11 & 12 & 13 & 14 & 15 & 16 \\
\hline$\chi\left(P_{n}\right)$ & 2 & 3 & 3 & 4 & 4 & $4 ?$ & $4 ?$ & $4 ?$ & $4 ?$ & $4 ?$ & $4 ?$ & $4 ?$ & $4 ?$ & $4 ?$ \\
\hline
\end{tabular}

\section{Acknowledgment}

The research was supported by RFBR Grant 15-01-05867 and by the Slovenian Research Agency in the frame of the research project "On odd automorphism in vertex-transitive (di)graphs", 2016-2018. The author thanks Kristina Rogalskaya for a nice picture of a proper 3 -coloring of $P_{5}$ and Pavel Konstantinov for critical suggestions, which helped to improve the clarity of the manuscript. 


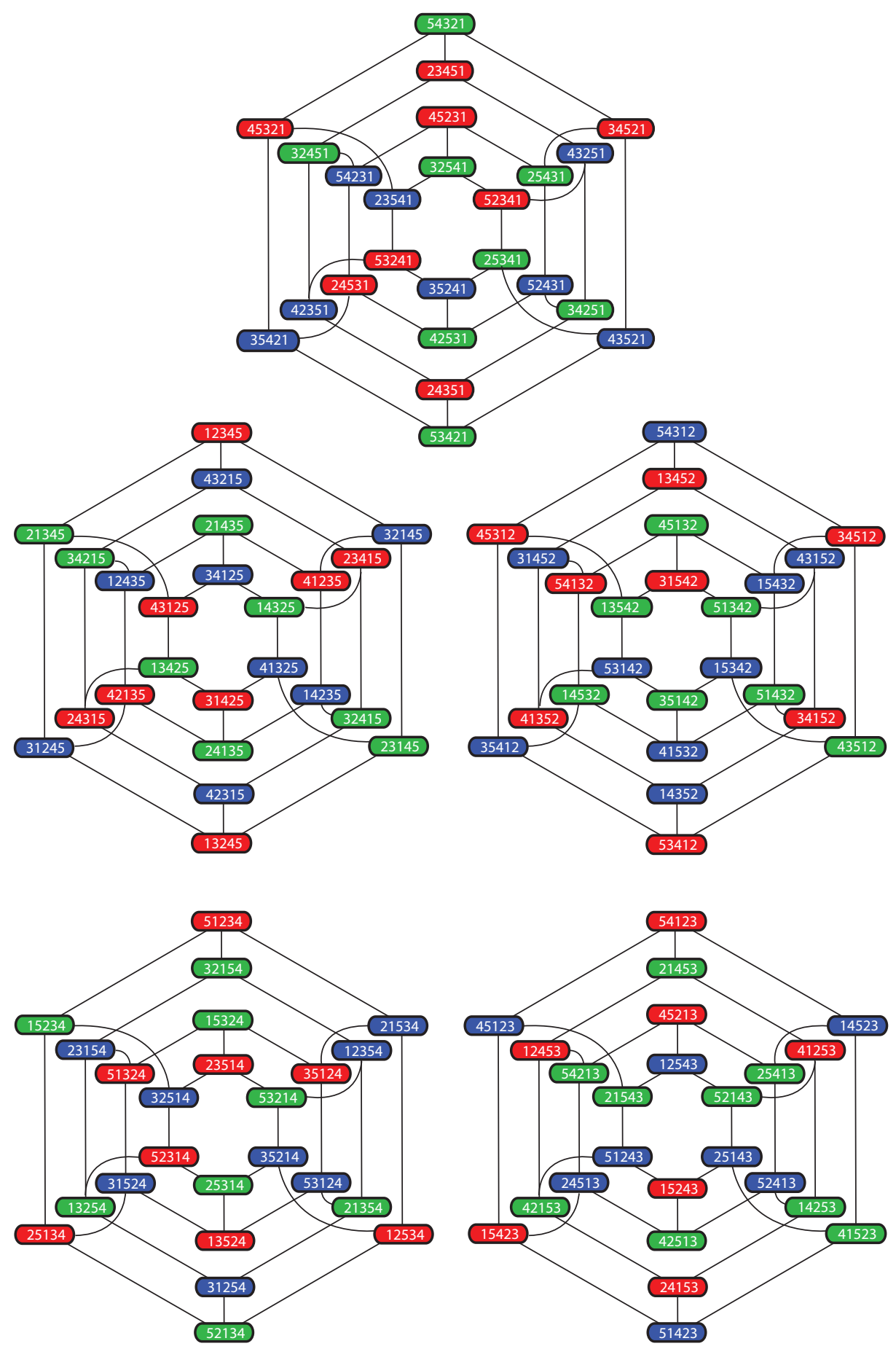

Appendix: Proper 3-coloring of $P_{5}$. 


\section{REFERENCES}

[1] O.V. Borodin and A.V. Kostochka, On an upper bound of a graph's chromatic number depending on the graph's degree and destiny, J. Combin. Theory. Ser. B. 23 (1977) 247-250. doi:10.1016/0095-8956(77)90037-5

[2] R.L. Brooks, On colouring the nodes of a network, Proc. Cambridge Phil. Soc. 37 (1941) 194-197. doi:10.1017/S030500410002168X

[3] P.A. Catlin, A bound on the chromatic number of a graph, Discrete Math. 22 (1978) 81-84. doi:10.1016/0012-365X(78)90049-3

[4] P.A. Catlin, Another bound on the chromatic number of a graph, Discrete Math. 24 (1978) 1-6. doi:10.1016/0012-365X(78)90167-X

[5] I.J. Dejter and O. Serra, Efficient dominating sets in Cayley graphs, Discrete Appl. Math. 129 (2003) 319-328. doi:10.1016/S0166-218X(02)00573-5

[6] H. Dweighter, E 2569 in: Elementary problems and solutions, Amer. Math. Monthly 82 (1975) 1010.

[7] A. Johansson, Asymptotic choice number for triangle free graphs, DIMACS Technical Report (1996) 91-95.

[8] A. Kanevsky and C. Feng, On the embedding of cycles in Pancake graphs, Parallel Comput. 21 (1995) 923-936. doi:10.1016/0167-8191(94)00096-S

[9] E. Konstantinova, On some structural properties of Star and Pancake graphs, Lecture Notes in Comput. Sci. 7777 (2013) 472-487.

doi:10.1007/978-3-642-36899-8_23

[10] E. Konstantinova and A. Medvedev, Independent even cycles in the Pancake graph and greedy Prefix-reversal Gray codes, Graphs Combin. 32 (2016) 1965-1978. doi:10.1007/s00373-016-1679-x

[11] J.J. Sheu, J.J.M. Tan and K.T. Chu, Cycle embedding in Pancake interconnection networks, in: Proc. 23rd Workshop on Combinatorial Mathematics and Computation Theory (Taiwan, 2006) 85-92.

[12] K. Qiu, Optimal broadcasting algorithms for multiple messages on the Star and Pancake graphs using minimum dominating sets, Congr. Numer. 181 (2006) 33-39.

[13] V.G. Vizing, Some unsolved problems in graph theory, Uspekhi Mat. Nauk 23 (1968) 117-134. doi:10.1070/rm1968v023n06abeh001252

Received 15 March 2016

Revised 12 July 2016

Accepted 12 July 2016 